\title{
改进的深度反投影网络红外图像超分辨率重建
}

\author{
林仁浦 $^{1}$, 张 力 $^{1}$, 马晨晖 ${ }^{1}$, 刘 轩 $^{1}$, 张 豪 $^{2}$ \\ (1. 火箭军工程大学, 陕西 西安 710025; 2. 31608 部队, 福建 厦门 361023)
}

\begin{abstract}
摘要: 深度反投影网络在可见光图像的超分辨率重建中具有优异的表现, 本文探索将深度反投影网络 应用到红外图像超分辨率重建中。针对红外图像对比度低、图像质量不高的特点, 在深度反投影网络 框架上作如下改进: 在上采样模块之前添加串联层, 将前一次的下采样输出和原始低分辨率预处理图 像串联作为上采样模块的输入, 以此提高网络获取图像高频信息的能力, 增强生成图像的细节信息。 实验结果证明, 本文算法较改进前能够得到细节更加丰富、视觉效果更加良好的红外超分辨率重建图 像。
\end{abstract}

关键词: 红外图像; 超分辨率; 深度反投影网络

中图分类号：TP183 文献标识码：A 文章编号：1001-8891(2020)09-0873-07

\section{Improved Super-resolution Reconstruction of Infrared Images Based on Deep Back-projection Networks}

\author{
LIN Renpu ${ }^{1}$, ZHANG $\mathrm{Li}^{1}$, MA Chenhui ${ }^{1}$, LIU Xuan ${ }^{1}$, ZHANG Hao ${ }^{2}$ \\ (1. Rocket Force University of Engineering, Xi'an 710025, China; 2. 31608 Troops, Xiamen 361023, China)
}

\begin{abstract}
Deep back-projection networks have excellent performance in the super-resolution reconstruction of visual images. This paper explores the application of deep back-projection networks to the super-resolution reconstruction of infrared images. In view of the characteristics of low infrared image contrast and low image quality, the following improvements were made in the framework of the deep back-projection network: adding a concatenation layer before the upsampling module, cascading the previous downsampling output and the original low-resolution preprocessed image as the input of the upsampling module. This was designed to improve the network's ability to obtain high-frequency information of the image and enhance the detail of the generated image. The experimental results proved that the proposed algorithm could create infrared super-resolution reconstructed images with richer details and improved visual effects.
\end{abstract}

Key words: infrared images, super-resolution, deep back-projection networks

\section{0 引言}

红外图像是利用探测器捕获物体红外辐射所呈 现出的图像, 自然界中不存在绝对零度的物体, 任何 物体都会向外辐射红外线。物体辐射红外线的强度受 物体的温度、材料、形状等影响, 物体辐射红外线强 度的差异, 在红外探测设备上得以表现, 进而将这种 差异用于区分成像物体。红外图像反映的是被观测物 体和背景之间的温度差, 这一特性使得红外图像通常 不受天候的影响, 因而红外图像常被用于夜间的监控 和侦察。受制于红外硬件系统的客观发展, 红外图像 在实际应用中都会涉及到分辨率较低的问题, 提高红
外图像的分辨率在遥感、目标检测识别等领域具有迫 切的应用需求 ${ }^{[1]}$ 。

当前, 图像的超分辨率重建技术是一个热点研究 方向, 超分辨率重建技术通常被分为 3 类: 基于插值 的算法 ${ }^{[2]}$ 、基于重建的算法 ${ }^{[3]}$ 和基于深度学习的算法 ${ }^{[4]}$ 。 基于插值的方法通常利用图像相邻像素之间的统计 特征处理图像, 这种方法虽然能够增强图像分辨率, 但图像的边缘会比较模糊。基于重建的算法主要用于 恢复图像的高频信息, 对于结构复杂的图像, 处理效 果较差。基于深度学习的算法通过对大量数据样本的 学习, 能够建立高分辨率图像和低分辨率图像之间的 非线性映射关系, 目前此类方法在超分辨率重建中取 
得了较好的应用效果。

Dong 等 ${ }^{[5]}$ 首次提出用于解决图像超分辨率重建 的卷积神经网络 SRCNN (Super-Resolution Convolutional Neural Networks), 该网络主要由 3 个 卷积层构成, 由于卷积操作无法对图像进行放大, 所 以在使用 SRCNN 网络时, 首先需要利用双三次插值 算法对图像进行预处理, 将低分辨率图像放大至目标 大小。之后 Dong 等又提出改进型算法 FSRCNN ${ }^{[6]}$ (Faster Super-Resolution Convolutional Neural Networks), 在 FSRCNN 结构中引入反卷积操作作为图像放大的手 段, 替代双三次插值的预处理。Kim 等 ${ }^{[7]}$ 提出用于图 像超分辨率的深度卷积神经网络 VDSR (Very Deep Convolutional Networks），该方法利用梯度剪裁来解 决由网络结构过深而引起的梯度消失和梯度爆炸。

Haris 等 ${ }^{[8]}$ 提出深度反投影网络 DBPN (Deep Back-Projection Networks）, 该网络包含相互依赖的 上-下采样模块, 通过这样的方式提供错误反馈机制, 同时利用深度级联的方式将不同的图像退化和超分 辨率组件包含在网络中, DBPN 相对于 SRCNN、 FSRCNN、VDSR 等算法在图像超分辨率重建质量上 有较明显的提升, 但在实际训练中 DBPN 从原始低分 辨率图像上只进行了一次信息获取, 一旦前面的上下 采样层丢失细节信息较多, 那么该网络的特征提取能 力就会显著下降。目前神经网络算法在可见光领域中 的超分辨率重建中取得了一定的技术性突破, 探究将 这类算法改进运用到红外图像的超分辨率重建中, 将 会推动红外图像成像技术的发展。

考虑到 DBPN 在可见光图像超分辨率重建算法 中相较其他图像重建算法效果更加优异, 本文探索在 DBPN 算法结构的基础上, 提出一种可以适合红外图 像超分辨率重建的方法。

\section{1 本文算法}

本文针对红外图像的特征, 在 DBPN 算法的基础 上提出改进型算法, 提高网络对红外图像高频信息的 获取能力。

\section{1 深度反投影网络}

深度反投影网络由 Haris 等人 ${ }^{[8]}$ 于 2018 年提出, 该网络结构包含数个串联的上下采样层, 通过不断地 对图片进行退化和超分辨率重建来提取图片中的空 间细节信息。深度反投影网络主要包括初始化特征提 取模块, 反投影模块, 以及重建模块, 具体框架结构, 如图 1 所示。改进型算法是在原先的算法基础上添加 了融合通道, 融合预处理图像和网络处理过程中的中 间变量。

\subsection{1 初始化特征提取模块}

初始化特征提取模块由两个卷积层组成, 其中第 一个卷积层的卷积核大小 kernel $=3$, 卷积操作的步长 stride $=1$, 零填充 padding $=1$, 经过该层卷积操作图 像的尺寸大小没有发生改变, 但是却可以根据实验需 求改变图像的通道数; 第二层卷积操作是一个卷积核 大小 kernel $=1$ 的卷积操作, 该层操作主要是修改图 像的通道数, 生成与原始图像相同通道数的图片。初 始化特征提取模块通过卷积操作提取图像中的特征 细节。

\subsection{2 反投影模块}

反投影模块由多个上下采样层相互连接组成, 这 种连接方式使得网络能够在不同深度获取 HR （High-Resolution）的特征, 同时上下采样层可以使用 不同类型的图像退化和超分辨率组件, 从而能够更加 丰富地获取图像细节信息。

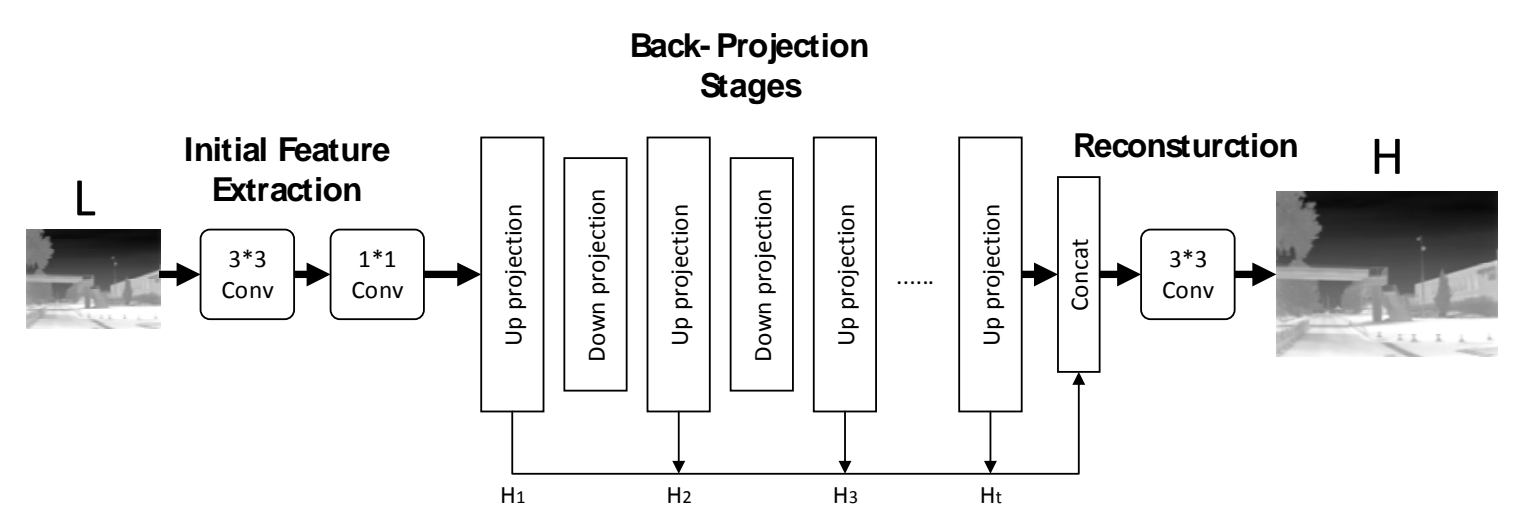

图 1 深度反投影网络结构图, 主要包括初始化特征提取、反投影和重建 3 个部分

Fig.1 Architecture of the deep back-projection networks, it mainly consists of initial feature extraction, back-projection stages and reconstruction module 
如图 2 所示, 深度反投影网络的向上反投影单元, 主要经过反卷积操作、卷积操作和反卷积操作, 具体 步骤如下:

放大:

$$
H_{t-1}^{0}=\left(L_{t-1} * p_{t-1}\right) \uparrow_{\mathrm{s}}
$$

缩小:

$$
L_{t-1}^{1}=\left(H_{t-1}^{0} * g_{t-1}\right) \downarrow_{\mathrm{s}}
$$

残差计算:

$$
e_{t-1}^{L}=L_{t-1}^{1}-L_{t-1}
$$

残差结果放大:

$$
H_{t-1}^{1}=\left(e_{t-1}^{L} * p_{t-1}\right) \uparrow_{\mathrm{s}}
$$

输出特征图:

$$
H_{t}=H_{t-1}^{0}+H_{t-1}^{1}
$$

式中: *表示卷积操作; $\uparrow_{\mathrm{s}}$ 和 $\downarrow_{\mathrm{s}}$ 分别代表上采样和下 采样操作; $p_{t^{-1}}$ 代表反卷积操作; $g_{t^{-1}}$ 代表卷积操作。

如图 3 所示, 深度反投影网络的向下反投影单元, 主要经过卷积操作、反卷积操作和卷积操作, 具体步 骤如下:

缩小:

$$
L_{t-1}^{0}=\left(H_{t-1} * g_{t-1}^{\prime}\right) \downarrow_{s}
$$

放大：

$$
H_{t-1}^{1}=\left(L_{t-1}^{0} * p_{t-1}^{\prime}\right) \uparrow_{\mathrm{s}}
$$

残差计算:

$$
e_{t-1}^{H}=H_{t-1}^{1}-H_{t-1}
$$

残差结果缩小:

$$
L_{t-1}^{1}=\left(e_{t-1}^{H} * g_{t-1}^{\prime}\right) \downarrow_{s}
$$

输出特征图:

$$
L_{t}=L_{t-1}^{0}+L_{t-1}^{1}
$$

式中: $p_{t^{-1}}{ }^{\prime}$ 代表反卷积操作; $g_{t-1}{ }^{\prime}$ 代表卷积操作。

1.1.3 重建模块

重建模块包含一个串联层和卷积层, 串联层将所 有上采样层的输出进行串联, 这使得网络能够充分利 用前面所有的高分辨率特征图, 最后通过卷积层来融 合所有特征图的特征信息, 并归一化图片的通道。

1.1.4 融合通道

如图 4 所示, 本文提出的改进算法在原算法的基 础上, 在上反投影单元之前添加串联层, 将经过预处 理的图像 Lpre 和下反投影单元的输出串联后输入到 上反投影单元, 通过这样的方式不断强化网络获取高 频信息的能力。反投影模块中的上下反投影单元采用 不同的 HR 和 LR（Low-Resolution）算法, 融合通道 为不同的上下反投影单元提供了细节信息更加丰富 的输入图像，由于不同 HR、LR 算法在图像纹理信息 获取能力不同, 引入融合通道能够结合不同 HR、LR 算法的特点, 图 5 为添加了融合通道的改进型算法模 型。

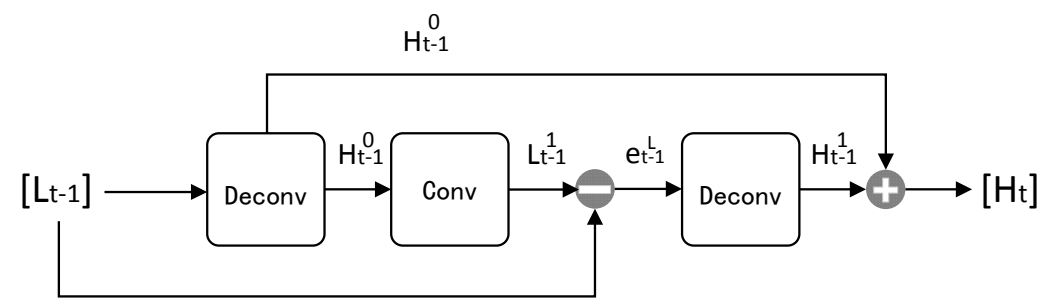

图 2 深度反投影网络的向上反投影单元 Fig.2 Up-projection unit in the DBPN

$\mathrm{Lt}^{0}-1$

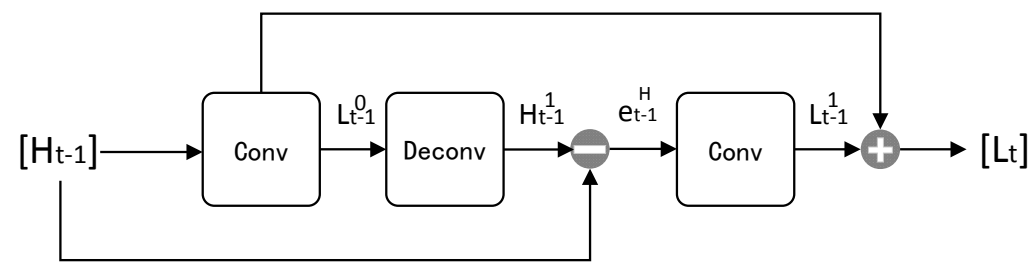

图 3 深度反投影网络中的向下反投影单元

Fig.3 Down-projection unit in the DBPN 


\section{2 实验}

\section{1 数据}

本文数据采用 CVC-09 以及 CVC-14 红外公开数 据集 ${ }^{[9]}$, 选用 CVC-09 数据集中的 7000 张红外图像作 为训练集, 选用 CVC-14 中的部分红外图像作为测试 集, 在进行训练前, 分别对训练集和测试集图像进行 下采样操作获得用于训练和测试的低分辨率数据集, 下采样因子为 4 。使用主观评价方法和峰值信噪比 (Peak Signal-to-Noise Ratio, PSNR) 、结构相似性 (Structural Similarity, SSIM) ${ }^{[10]} 3$ 种评价方法对本文 算法效果进行评估, 同时选用 Bicubic ${ }^{[11]} 、 S R C N N$ 和 DBPN 算法作为本文算法的对比算法。

\section{2 实验设置}

本文神经网络训练平台采用 pytorch, GPU 采用 GTX1080ti, 设定的学习速率为 0.0001 , 采用 Adam 模型优化, 迭代次数 50000 次。

\section{3 质量评价标准}

图像质量评价标准是衡量超分辨率图像重建算 法的重要指标, 目前图像质量评价标准主要分为客观 评价方法与主观评价方法。客观评价方法是通过设计 系统的算法, 依据图像的客观数据衡量超分辨率图像 的质量。主观评价方法是通过人眼观测图像, 并对图 像质量进行评判的方法。

\section{4 实验结果讨论}

实验选用了 CVC-14 中的图片作为测试集, 在网 络模型放大因子为 4 的基础上, 将本文算法的结果与 Bicubic、SRCNN、DBPN 的重建结果进行比较, 并利 用主观和客观两类评价指标进行对比。

\subsection{1 主观评价效果}

我们从实验数据中选取了 4 张图片, 在 4 倍放大 因子的基础上, 对比不同算法的实际重建效果, 具体 情况如图 6、图 7、图 8、图 9 所示。

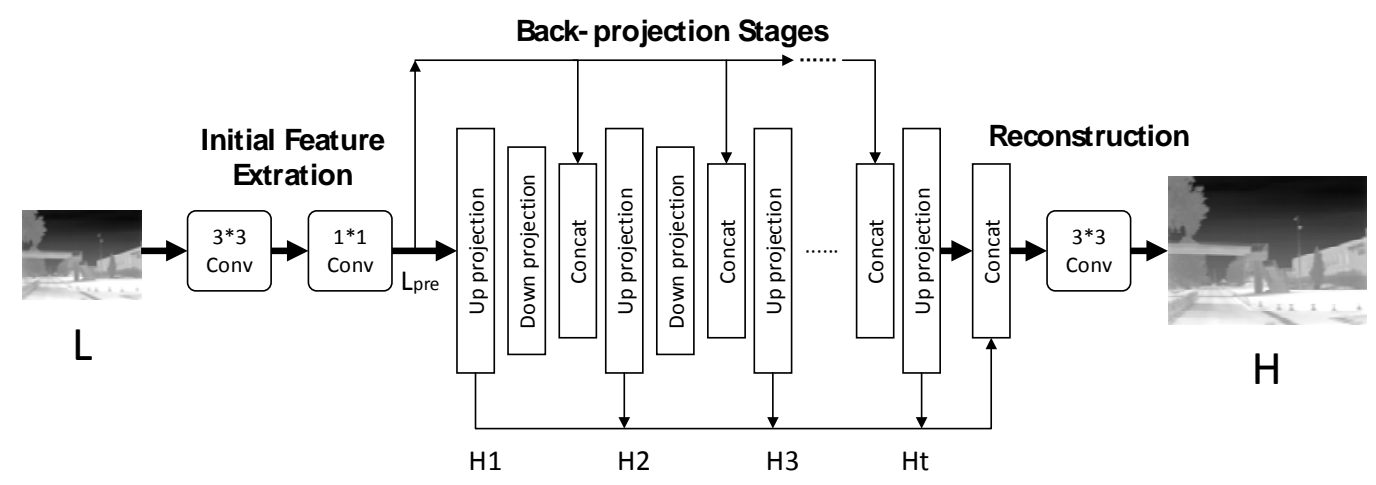

图 4 改进后的深度反投影网络 Fig.4 Improved deep back-projection network

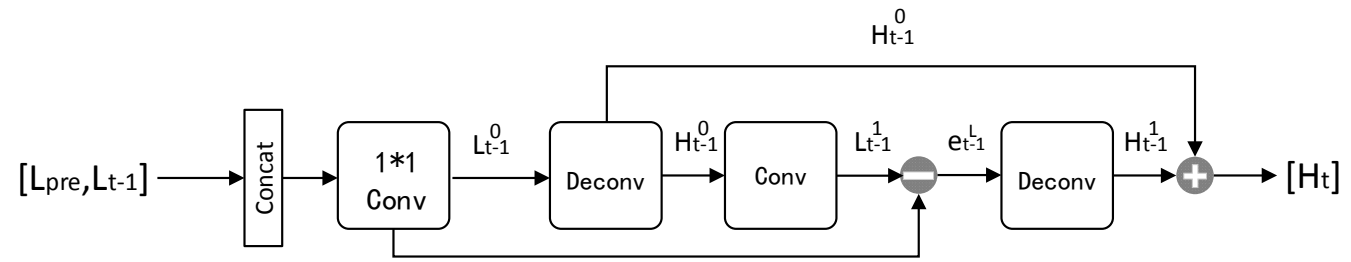

图 5 改进后的上采样层

Fig.5 Improved up-projection unit

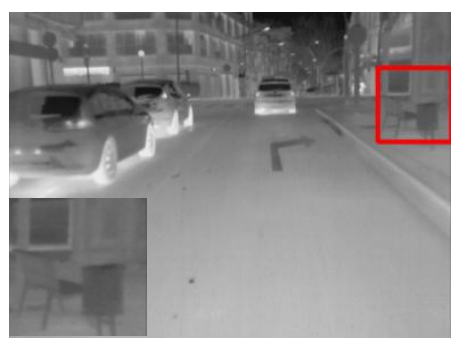

(a) 原始 HR 图像

(a) Original HR picture

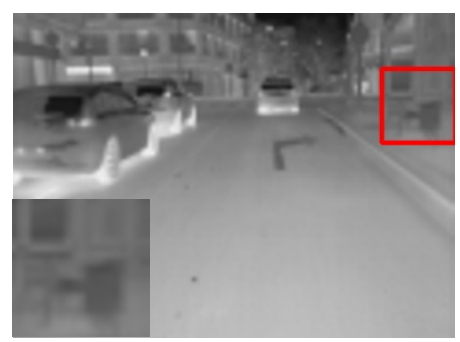

(b) Bicubic 算法

(b) Bicubic

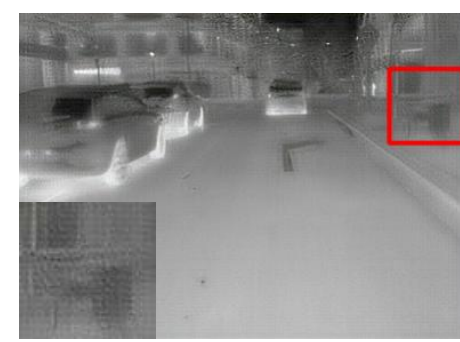

(c) SRCNN 算法

(c) SRCNN 


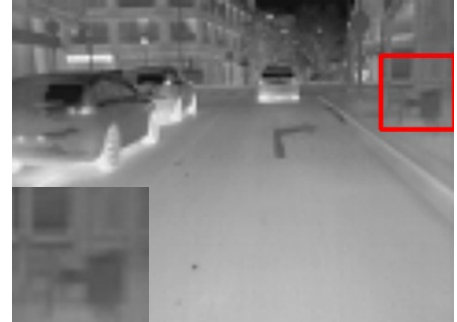

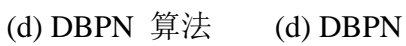

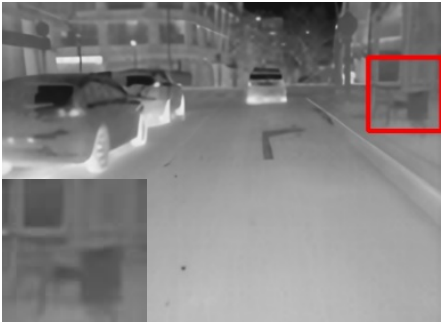

(e) 本文算法 (e) Proposed

图 6 行车图像使用不同超分辨率重建方法的重建结果对比

Fig.6 Comparison of reconstruction results of driving images by different super-resolution reconstruction methods

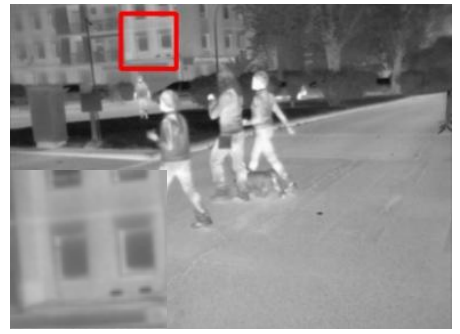

(a) 原始 HR 图像 (a) Original HR picture

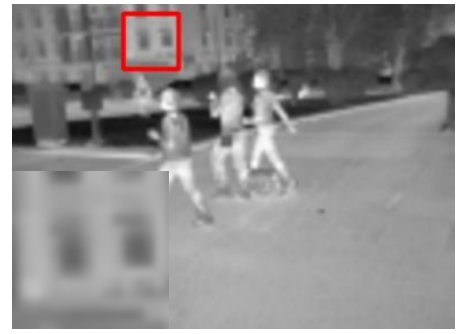

(b) Bicubic 算法

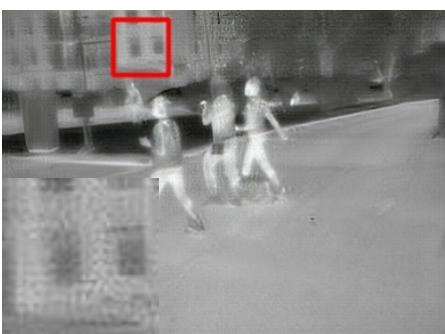

(c) SRCNN 算法

(c) SRCNN

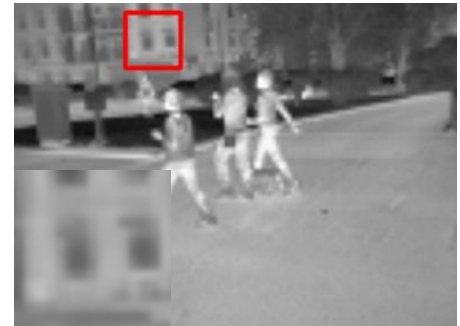

(d) DBPN 算法 (d) DBPN

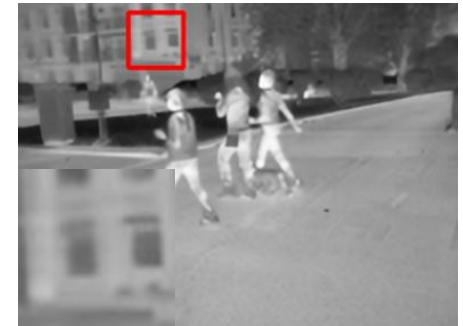

(e) 本文算法

(e) Proposed

图 7 行人图像使用不同超分辨率方法的重建结果对比

Fig.7 Comparison of pedestrian images by different super-resolution methods

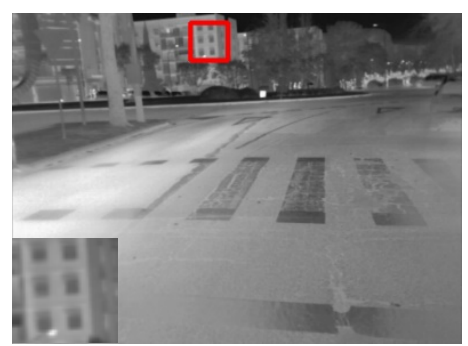

(a) 原始 HR 图像

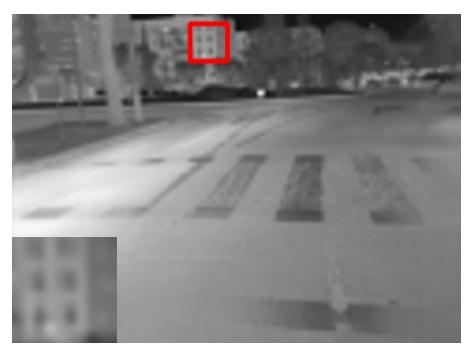

(b) Bicubic 算法

(a) Original HR picture

(b) Bicubic

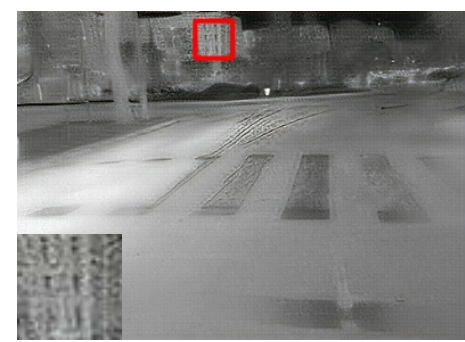

(c) SRCNN 算法

(c) SRCNN

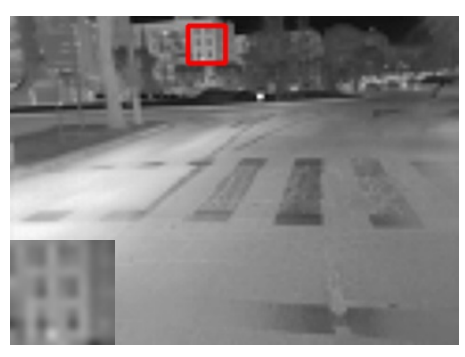

(d) DBPN 算法

(d) DBPN

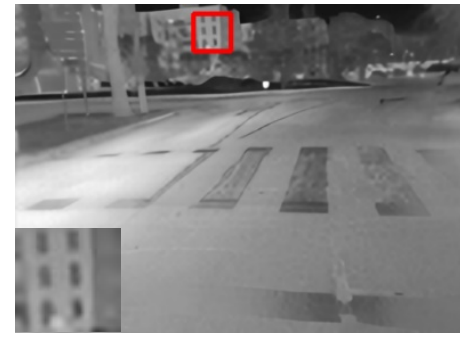

(e) 本文算法 (e) Proposed

图 8 斑马线图像使用不同超分辨率方法的重建结果对比

Fig.8 Comparison of zebra crossing images by different super-resolution methods 


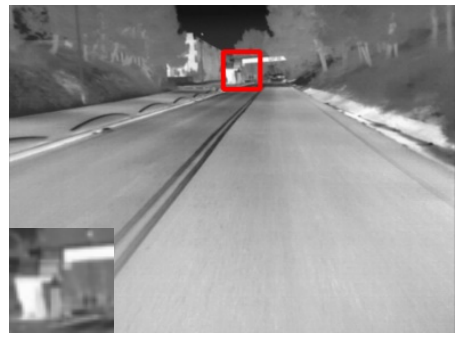

（a）原始 HR 图像 (a) Original HR picture

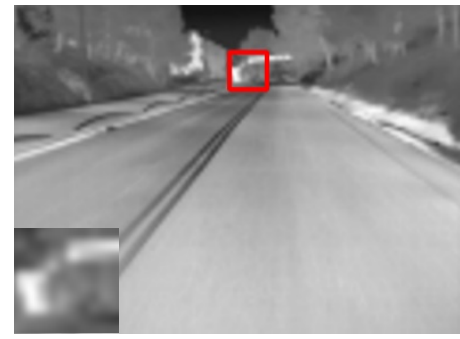

(b) Bicubic 算法 (b) Bicubic

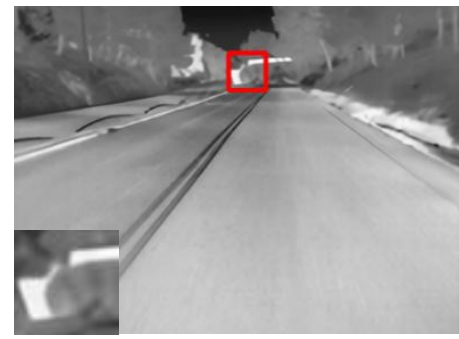

(e) 本文算法

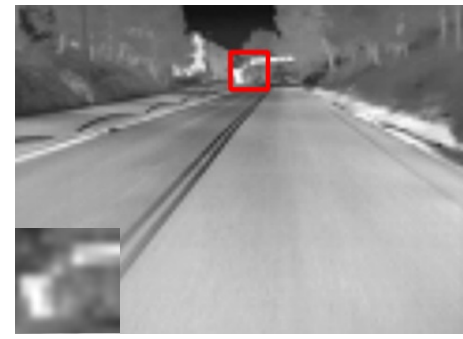

(c) SRCNN 算法

(c) SRCNN

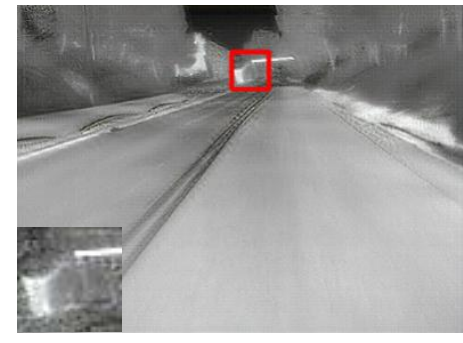

(d) DBPN 算法

(d) DBPN

图 9 马路图像使用不同超分辨率方法的重建结果对比

Fig.9 Comparison of road images by different super-resolution methods

图 6 表示的是行驶中汽车的街景图片, 对比本文 提出的算法和其他算法, 放大街景图片中的长登, 本 文所提算法能够清晰地还原出长登的细节, 从细节对 比来看, 本文算法相比其他算法具有一定的优势; 图 7 表示的是街边的行人图片, 放大街边建筑物的细节 后进行观察对比, 可以看出本文提出的算法在纹理细 节上更加丰富; 图 8 拍摄的是斑马线图片, 截取图片 中的建筑物细节, 可以发现本文算法能够比较逼真的 还原原始图像的细节; 图 9 表示的是行驶过程中拍摄 的马路图片, 放大远处的物体, 本文提出的算法能够 较平滑地还原物体细节。因此, 我们可以推断本文算 法在与 Bicubic、SRCNN、DBPN 等算法相比, 在图 像超分辨率重建方面具有明显的优势。

\subsection{2 客观评价效果}

本文采用的客观图像指标有 PSNR 和 SSIM。峰 值信噪比是对比两幅图像对应像素之间的误差, 通过 误差值判断图像的失真程度, 峰值信噪比的数值越大 表明生成图像越接近原始高分辨率图像, 图像的重建 质量越好。结构相似度用来衡量图像的相似程度, 该 指标数值越接近 1 , 则表明生成的超分辨率图像越接 近原始高分辨率图像, 超分辨率重建效果越好。

在 4 倍放大因子的条件下, 选用 CVC-14 中的 4 张图片, 对比本文算法和 Bicubic、SRCNN、DBPN 在 PSNR 和 SSIM 标准下的重建效果。如表 1 和表 2 所示, 本文算法在 PSNR 和 SSIM 评价标准下都能够 取得优于其它算法的实验结果, 因此可以证明本文算
法要优于其它算法。

表 1 不同重建方法的 PSNR 评价效果对比

Table 1 Comparison of PSNR values for different super-resolution methods

\begin{tabular}{lllll}
\hline Images & Bicubic & SRCNN & DBPN & Proposed \\
\hline Car & 29.08 & 30.06 & 32.59 & 33.55 \\
Pedestrian & 27.26 & 27.82 & 32.89 & 33.18 \\
Zebra Crossing & 26.35 & 28.22 & 30.32 & 30.83 \\
Road & 26.87 & 27.86 & 30.55 & 31.17 \\
\hline
\end{tabular}

表 2 不同重建方法的 SSIM 评价效果对比

Table 2 Comparison of SSIM values for different

\begin{tabular}{lllll}
\multicolumn{5}{c}{ super-resolution methods } \\
\hline Images & Bicubic & SRCNN & DBPN & Proposed \\
\hline Car & 0.8527 & 0.8611 & 0.8728 & 0.8995 \\
Pedestrian & 0.8465 & 0.8621 & 0.8697 & 0.8812 \\
Zebra Crossing & 0.8856 & 0.8912 & 0.9011 & 0.9125 \\
Road & 0.8952 & 0.9013 & 0.9133 & 0.9265 \\
\hline
\end{tabular}

\section{3 结论}

本文通过改进深度反投影网络算法, 获得了一种 可以应用到红外图像超分辨率重建的模型。在 DBPN 的网络模型中引入融合通道, 提高了上采样层对高频 信息的获取能力, 较原始网络结构能够获取更丰富的 纹理信息。实验结果表明该方法能够重建出细节信息 丰富的红外图像, 具有较好的视觉感知效果。最后通 
过主观和客观图像质量评估方法, 证明了本文改进算 法在重建红外图像上的实际效果。

\section{参考文献:}

[1] DONG W, SHI G, LI X, et al. Image reconstruction with locally adaptive sparsity and nonlocal robust regularization[J]. Image Communication, 2012, 27(10): 1109-1122.

[2] YANG H, HUANG Z. Fast image interpolation algorithm based on gradient[J]. Journal of Computer Applications, 2013, 32(10): 2821-2823.

[3] Papyan V, Elad M. Multi-Scale Patch-Based Image Restoration[J]. IEEE Transactions on Image Processing, 2015, 25: 249-261.

[4] 王威, 张彤, 王新. 用于图像超分辨率重构的深度学习方法综述 [J]. 小型微型计算机系统, 2019, 40(9): 1891-1896.

WANG Wei, ZHANG Tong, WANG Xin. Survey of Single Image Super-resolution Based on Deep Learning[J]. Journal of Chinese Computer Systems, 2019, 40(9): 1891-1896.

[5] DONG C , LOY C C , HE K , et al. Image Super-Resolution Using Deep Convolutional Networks[J]. IEEE Trans Pattern Anal Mach Intell, 2016,
38(2): 295-307.

[6] DONG C, LOY C C, TANG X. Accelerating the super-resolution convolutional neural network[C]//European Conference on Computer Vision, 2016: 391-407.

[7] KIM J, LEE J K, LEE K M. Accurate image super-resolution using very deep convolutional networks[C]//IEEE Conference on Computer Vision and Pattern Recognition, 2016: 1646-1654.

[8] Haris M, Shakhnarovich G, Ukita N. Deep Back-Projection Networks for Super-Resolution[C]//IEEE/CVF Conference on Computer Vision and Pattern Recognition, 2018: 1664-1673.

[9] SOCARRAS Y, RAMOS S, VAZQUEZ D, et al. Adapting Pedestrian Detection from Synthetic to Far Infrared Images[C]//ICCV - Workshop on Visual Domain Adaptation and Dataset Bias, 2013: 1-3.

[10] WANG Z, BOVIK A C, SHEIKH H R, et al. Image quality assessment: From error visibility to structural similarity[J]. IEEE Transactions on Image Processing, 2004, 13(4): 600-612.

[11] ZHANG L , WU X. An edge-guided image interpolation algorithm via directional filtering and data fusion[J]. IEEE Trans Image Process, 2006, 15(8): 2226-2238. 